\title{
Compressive Sensing Application in Large Scale Ultrasound Medicine Imaging
}

\author{
Ying $\mathrm{Li}^{1}$, Siyuan Wen ${ }^{2}$ \\ ${ }^{1}$ Department of Computer Science, Shandong Xiehe University, Jinan, 250107, China \\ ${ }^{2}$ School of Management Science and Engineering, Shandong University of Finance and \\ Economic, Jinan, 250014, China
}

Keywords: Compressed sensing, Ultrasound medicine imaging, Plane wave

\begin{abstract}
We use a compressed sensing method to store and recover the large-scale ultrasound medicine radio-frequency (RF) signals generated from plane wave (PW). We investigate performance of reconstruction on different ultrasound post-beamformed data when performing compressed sensing with fewer measurement data. Reconstruction is performed using convex optimization algorithm. For a single channel sequence, the iteration processing is fast even not more than 20 times. RF data from Field II simulation of a phantoms and the plane-wave imaging challenge in medical ultrasound (PICMUS) data sets. Using the 50\% measurement data, we get a closed approximate channel data. In term of mean-square-error, reconstruction on RF data performs well.
\end{abstract}

\section{Introduction}

Ultrasound imaging uses high frequency sound waves and their echoes to form an image of tissue to help diagnose the diseases in clinical application. It has been proven to be a high safe, cost effective, convenience and real-time modality for imaging soft tissue.

The conventional ultrasound imaging is called B-mode imaging in which the radio frequency (RF) signals are acquired after sending focused beams along one direction sequentially in time. Synthetic Transmit Aperture (STA) imaging is a method to solve the disadvantages of B-mode imaging. In STA, all the elements receive the backscattered RF signals using delay-and-sum algorithm to form a high-resolution image. In late 1990s and early 2000s, Fink et al. improved the idea of Plane Wave insonification to achieve ultrafast frame rates ( $>5000 \mathrm{fps}$ ). PW ultrasound imaging has been developed in the field of medical ultrasound imaging [1,2]. At each transmit event, an ultrasound wave was transformed from the whole array aperture and received by the whole elements. The transmit-receive process is repeated with a different steering to yield a series of low-resolution image (LRI), which has no transmit focusing. The combination of LRI gives to a high-resolution image. For practical use, the classical Nyquist sampling frequency usually results in huge size of sampled signal, which will hinder data storage and transfer. The compressed sensing (CS) theory indicates that if a signal is sparse or compressible, it can be recovered from limited number of projections with high probability. CS has attracted many attentions from the related fields since it was proposed, In the field of medical imaging, it has been applied to magnetic resonance [3], CT and also ultrasound imaging [4]. In this study, we try to accurately reconstruct the object from limited plane wave channel signals based on compressed sensing. The prosed method can not only save scanning time to reduce the distortion by respiratory movement, but also can reduce cost and complexity.

\section{Plane Wave Imaging and Compressed Sensing Method}

Plane Wave ultrasound imaging use synthetic aperture image reconstruction. The wave front is generated by interference of individual spherical waves produced by each array element. A delay d is required to be applied to the transmitting element. 


\subsection{The Principle of Plane Wave Imaging}

The principle of plane wave focusing relies on synthesizing the transmit focus by emitting a number of steered plane waves into the tissue. For each transmit-receive event, the travelling time to the point $(\mathrm{x}, \mathrm{z})$ and back to transducer element depends on the inclination angle $\alpha$ of transmission and the speed of the sound in the medium, as shown in Figure 1.

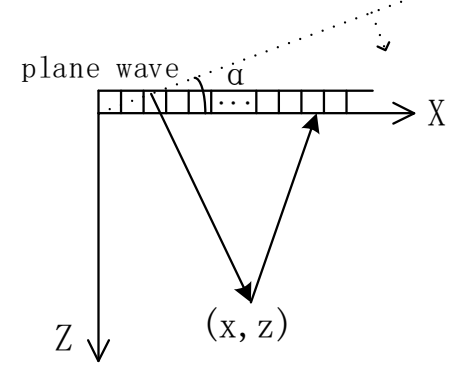

Fig.1 Plane Wave Imaging.

The time required for the transmitted wave to reach the point $(\mathrm{x}, \mathrm{z})$ is:

$$
t_{\text {transmit }}(\alpha, x, z)=\frac{z \cos \alpha+x \sin \alpha}{c}
$$

The time required to receive a wave from point $(\mathrm{x}, \mathrm{z})$ to the transducer element located $\mathrm{at}_{1}$ is:

$$
t_{\text {receive }}\left(x_{1}, x, z\right)=\frac{\sqrt{z^{2}+\left(x-x_{1}\right)^{2}}}{c}
$$

Therefore, the total time for a PW insonification is:

$$
t\left(\alpha, x_{1}, x, z\right)=t_{\text {transmit }}(\alpha, x, z)+t_{\text {receive }}\left(x_{1}, x, z\right)
$$

$c$ is the speed of the wave travelling in the medium. In this paper, Field II simulation software [5] was used to simulate the PW RF signal in two dimensions.

\subsection{Compressed Sensing Theory}

In compressed sensing theory [6], a sparse vector $x \in R^{n}$ can be recovered from a small number of linear measurements $b=A x, b \in R^{k}, k<n$ (or $b=A x+e$ )when there is measurement noise) by solving a convex program. $A$ is a known random $k \times n$ "sampling" matrix. To solve such kind of convex problem, quit a few of algorithms has been presented. such as Orthogonal Matching Pursuit(OMP), Primal-Dual Basis Pursuit(BP), Gradient Projection Sparse Representation, Bregman, Nesterov's Algorithm (NESTA), etc. Consider the characteristics of high dynamic range, large scale of plane wave ultrasound signals, NESTA performs well. By assuming that $x$ is sparse, the reconstruction problem can be recast as following optimization problem,

$$
\min \lambda\|x\|_{1}+\frac{1}{2}\|b-A x\|_{2}^{2}
$$

Assuming $x^{*}$ is the optimal solution, using NESTA algorithm it can be computed by iteration method. For kth iteration, $y_{k}, z_{k}$ can be compute using the Lagrangian solution under the Karush-kuhn-Tucker(KKT) condition.

$$
\begin{aligned}
& y_{k}=x_{k}-L^{-1} \nabla f\left(x_{k}\right) \\
& z_{k}=x_{0}-L^{-1} \sum_{i=1}^{k} \alpha_{i} \nabla f\left(x_{k}\right)^{T} \\
& x_{k+1}=\tau_{k} z_{k}+\left(1-\tau_{k}\right) y_{k}
\end{aligned}
$$


Here $\tau_{k}, \alpha_{k}$ affect the speed of convergency.

$$
\tau_{k}=\frac{2}{k+3}, \alpha_{k}=\frac{1}{2(k+1)}
$$

\subsection{Pw Ultrasound Pulse-Echo Signal Reconstruction}

In plane wave ultrasound imaging, $x$ is actually the collections of the echoes backscattered by all the scatters in the image area. it is sparse in Fourier domain. CS shows that the signal can have a sparse representation in a given basis and that the measurements are incoherent with that basis [7]. Here, we use the fast Walsh-Hadamard transform to get the sparse representation of down-sampled RF signal.

$$
x=\psi u
$$

Where $\mathrm{u}$ is the sparse transform coefficient. Using (2) in (1), we get

$$
y=A u+e
$$

Where $A_{k \times n}=\phi \psi$ is the measurement matrix, $x \in R^{n}$ is the signal sensed, $\phi_{k \times n}$ is the sample scheme, is the signal measured and e is the noise. And the pule-echo has a very large range dynamic, sometimes even more than $100 \mathrm{db}$. For very weak signal, the NAESTA will be failed. Here, we normalized the channel RF data before under-sampling so it can perform well.

\section{Results and Discussion}

\subsection{Results on Simulated Phantom}

We use Field II program to simulated raw RF signals received by a 128 elements linear array probe. The probe transmits a single plane-wave pulse and senses a $20 \mathrm{~mm}$-by- $110 \mathrm{~mm}$ area. The phantom contains 5 point targets separated by $5 \mathrm{~mm}$. Their amplitudes dynamic range is about $148 \mathrm{~dB}$. The data were first down sampled in the axial dimension by a factor 2 . Parameters are given in Table 1.

Figure 2 are the plane wave images for the simulated point-target phantom. To quantify the recover error, the normalized root-mean-square error between the full channel data and its recovered version from CS could be calculated. The results show that when using CS recovery, the obtained imaging performance is similar.

Table 1 Simulation Parameters

\begin{tabular}{|l|l|}
\hline Parameter & Value \\
\hline $\mathrm{F}_{0}$ & $5.133 \mathrm{MHz}$ \\
\hline Fs & $100 \mathrm{MHz}$ \\
\hline Width & $0.3 \mathrm{~mm}$ \\
\hline Hight & $5 \mathrm{~mm}$ \\
\hline Kerf & $0.03 \mathrm{~mm}$ \\
\hline Number of elements & 128 \\
\hline Number of alpha & 15 \\
\hline
\end{tabular}




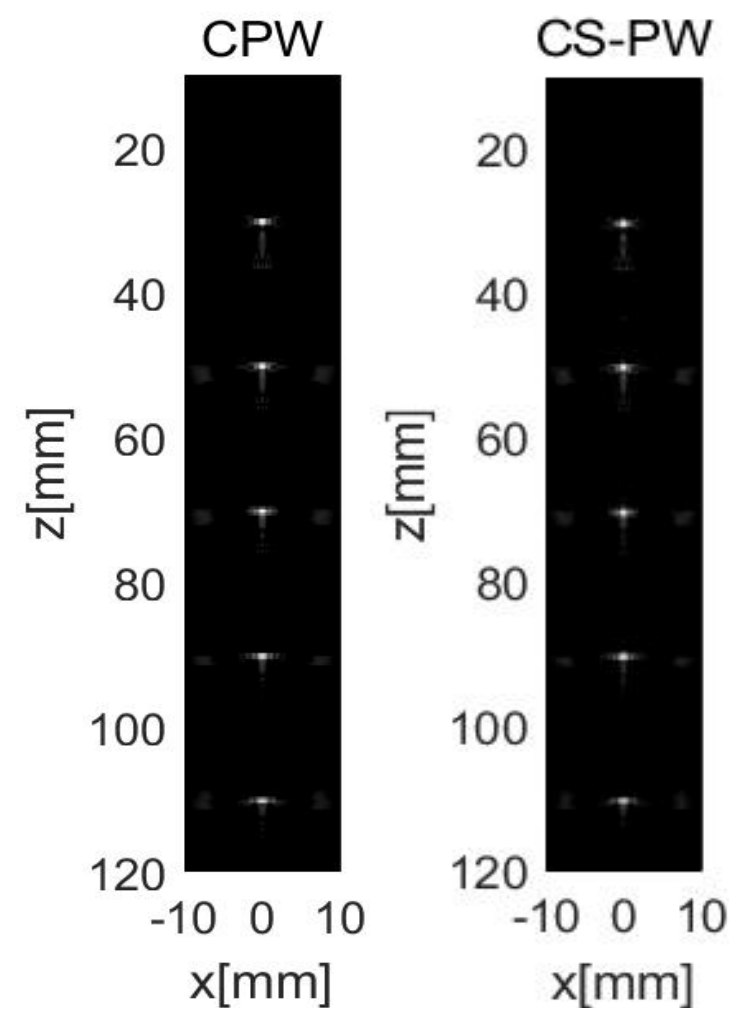

Fig.2 Plane Wave Imaging for the Simulated Point-Target Phantom

\subsection{Results on Experiments}

We apply our method on data from the PICMUS challenge dataset. Figure 3 imaging from a 75 plane-wave sequence recorded with a Verasonics Vantage 256 research scanner and a L11 probe (Verasonics Inc., Redmond, WA). The dataset was recorded on the carotid artery of a volunteer.

The coherent plane wave (CPW) imaging used all the backscattered signals from receiving elements. Whereas, using the compressed sensing plane wave (CS-PW) imaging method as we proposed only need half of the signals of the CPW imaging and almost no loss in quality of imaging.
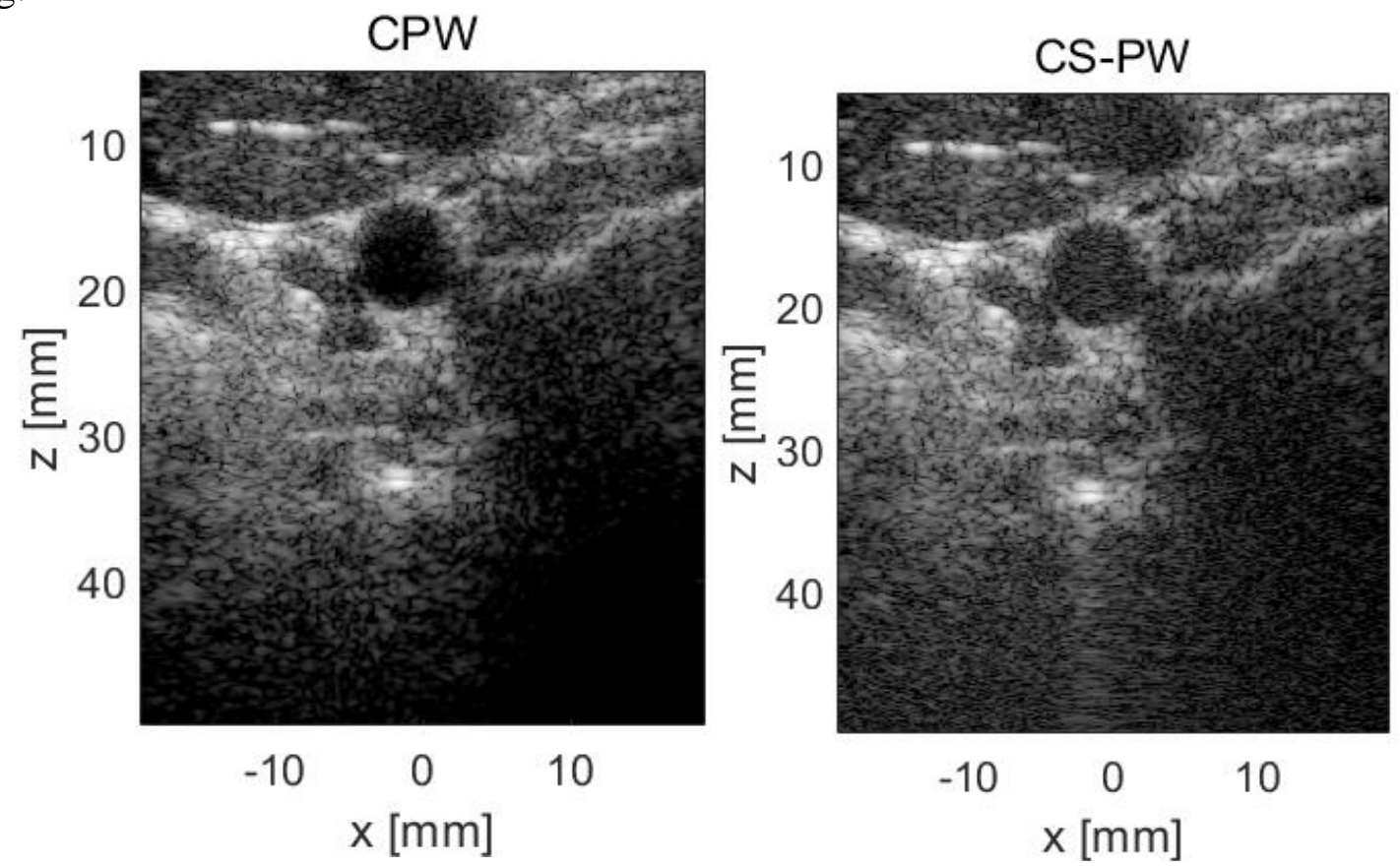

Fig.3 Plane Wave Imaging of the Carotid Artery 


\section{Conclusion}

This paper proposed a plane wave imaging based on CS method used in large scale and high dynamic range ultrasound medical imaging. Phantom simulation and PICMUS experiments are performed, the results demonstrate that CS-PW is effective in increasing the frame rate while keeping the high resolution.

\section{References}

[1] Jespersen, S.K., Wilhjelm, J.E. and Sillesen, H. (1998). Multi-angle Compound Imaging. Ultrasound Imaging, vol. 20, pp.81-102.

[2] Entrekin, R.R., Porter, B.A., Sillesen, H.H., et.al. (2001). Real-time Spatial Compound Imaging: Application to Breast, Vascular, and Musculoskeletal Ultrasound. Semin Ultrasound CT MR, vol. 22, pp.50-64.

[3] Lusfig, M.,Donoho, D.,Pauly, J. M. (2007). Sparse MRI: The Application of Compressed Sensing for Rapid MR Imaging.Magnetic Resonance in Medicine. vol.58, no.6, pp.1182-1195.

[4] Tur, R., Eldar Y.C.,Friedman, Z. (2011). Innovation Rate Sampling of Pulse Streams with Application to Ultrasound Imaging.IEEE Transactions on Signal Processing. vol. 59, no.4, pp.1827-1842.

[5] Jensen, J. A. Field: A program for Simulating Ultrasound Systems. (1991). Medical \& Biological Engineering \& Computing. vol. 34, pp.351-353.

[6] Emmanuel J.C., Tao T.(2006). Near-optimal Signal Recovery from Random Projections: Universal Encoding Strategies? IEEE Transactions on Information Theory. vol.52, no.12, pp.5406-5425.

[7] Emmanuel C., Romberg, J. (2007). Sparsity and Incoherence in Compressive Sampling. Inverse Problems. vol.23, no.3, pp.969-985. 